\title{
The UK's pandemic influenza research portfolio: a model for future research on emerging infections
}

Professor Colin R Simpson PhD ${ }^{\mathrm{a} b}$, *, Mr Dan Beever MPH ${ }^{\mathrm{c}}$, Dr Kirsty Challen PhD ${ }^{\mathrm{d}}$, Dr Daniela De Angelis PhD ${ }^{\mathrm{e}}$, Ms Ellen Fragaszy MSc ${ }^{\text {f g }}$, Professor Steve Goodacre $\mathrm{PhD}^{\mathrm{h}}$, Professor Andrew Hayward MD ${ }^{\mathrm{f}}$, Professor Wei Shen Lim DM ${ }^{\mathrm{j}}$, Dr G James Rubin PhD ${ }^{\mathrm{k}}$, Professor Malcolm G Semple PhD ${ }^{1}$, Professor Marian Knight DPhil ${ }^{\mathrm{m}}$, on behalf of the NIHR hibernated influenza studies collaborative group

a Usher Institute, The University of Edinburgh, Edinburgh, UK.

b Faculty of Health, Victoria University of Wellington, Wellington, New Zealand.

c. Clinical Trials Research Unit, School of Health and Related Research (ScHARR), University of Sheffield, Sheffield, UK

d. Lancashire Teaching Hospitals NHS Trust and Honorary Senior Lecturer, Centre for Urgent and Emergency Care Research, University of Sheffield, UK

e MRC Biostatistics Unit, University of Cambridge

f Centre for Public Health Data Science, Institute of Health Informatics, University College London, London, UK

g Department of Infectious Disease Epidemiology, London School of Hygiene and Tropical Medicine, London, UK

h Centre for Urgent and Emergency Care Research, School of Health and Related Research (ScHARR), University of Sheffield

i Institute of Epidemiology and Health Care, University College London, London, UK

j Nottingham University Hospitals NHS Trust, Nottingham, UK

k Department of Psychological Medicine, Weston Education Centre, King's College London, London, UK

1 Institute of Translational Medicine, University of Liverpool, UK

m National Perinatal Epidemiology Unit, University of Oxford, Oxford, UK

* Corresponding author

NIHR hibernated influenza studies collaborative group:

Marian Knight, Colin R Simpson, Dan Beever, Kirsty Challen, Daniela De Angelis, Ellen Fragaszy, Steve Goodacre, Andrew Hayward, Wei Shen Lim, Garry Meakin, G James Rubin, Malcolm G Semple, Helen Walters. 


\section{Summary}

The 2009 A/H1N1 influenza pandemic was responsible for considerable global morbidity and mortality. In 2009, UK funders, including the National Institute for Health Research (NIHR) rapidly funded and activated a number of research studies to inform clinical and public health actions. However, even with accelerated processes, some studies were completed too late for their results to have an early and significant impact on clinical care. This was in contrast to a study funded separately in 2008, which was published within a matter of weeks after the first two cases of 2009 A/H1N1 influenza virus infection were detected in the UK. In recognition of the impact of the NIHR-funded 2009 A/H1N1 influenza work and following reflection on the inherent delays in calling for research proposals, assessing, funding, and setting up the subsequent projects, including obtaining relevant ethical and regulatory approvals, the NIHR funded a second wave of studies in 2012. Our portfolio of projects have now been set-up (relevant permissions put in place, arrangements made for data collection) and pilot tested where relevant. All studies are now in 'hibernation' - that is, they have been put on standby mode in a maintenance-only state to await activation in the event of a pandemic being declared. In this thought piece, we describe the projects that were set up, the challenges of putting these projects into hibernation, on-going activities to maintain readiness for activation, and discuss how we think about planning research for a range of major incidents (e.g. other emerging infectious diseases, chemical, biological, radiological or nuclear (CBRN) risks, extreme weather events or industrial accidents). 


\section{Introduction}

In response to the $2009 \mathrm{~A} / \mathrm{H} 1 \mathrm{~N} 1$ influenza pandemic, the UK's funders, including the National Institute for Health Research (NIHR) rapidly sponsored and activated a number of research studies to inform clinical and public health actions (Table 1). That programme demonstrated the ability of the NIHR to commission, fund and deliver clinically relevant research under challenging circumstances [1]. However, even with accelerated processes, some studies were completed too late for their results to have an immediate significant impact on clinical care [24]. This experience was shared by major research networks in other countries [5]. In contrast, studies which had been funded separately, either prior to the emergence of the pandemic [6] or via Department of Health in England were able to provide timely reports on their results [7].

In recognition of the impact of the NIHR-funded 2009 A/H1N1 influenza work, and following reflection on the inherent delays in calling for research proposals, assessing, funding and setting up the subsequent projects, including obtaining relevant ethical and regulatory approvals, the NIHR funded a second wave of studies in 2012. These projects were set up (relevant permissions put in place, arrangements made for data collection) and pilot tested where relevant. All of the studies are being maintained in a state of readiness or 'hibernation' in that they are on standby mode in a maintenance-only state to await activation in the event of a pandemic being declared. Other organisations have subsequently recognised the need for better planning of research for future pandemics [8].

\section{The NIHR CRN portfolio of pandemic influenza studies}

The portfolio of studies (Table 1) cover key pathways of healthcare for influenza illness, including: surveillance, primary prevention (vaccination), triage (both in the community and at the hospital front door) and clinical management (therapeutics). 
The population-level susceptibility, severity and spread of pandemic influenza study (PIPS) has developed the Health Survey for England as a tool for rapid population based serological surveys of influenza infection and disease [9]. During the first two waves of a pandemic, the study will assess the public's susceptibility to and spread of the novel virus both geographically and by age. By combining our estimated number of infections in the population with estimates of the numbers of hospitalisations and deaths, we can quantify the proportion of infections leading to these outcomes - a key measure of pandemic severity.

The Flu Telephone Survey Template study (FluTEST) has been funded to develop rapid turnaround telephone surveys, for use in monitoring behaviour across the general population and identifying ways of improving how we communicate our public health advice [10]. How the public behave during any future pandemic will play a crucial role in how quickly influenza spreads in our community. Understanding whether the public are adhering to public health recommendations e.g. washing hands, being vaccinated and using health services appropriately, and if not, why not, is essential information [6][10-11]

The EAVE (Early estimation of pandemic influenza Antiviral and Vaccine Effectiveness) project has created a new sentinel system linking primary care data to RT-PCR swabs, stored serology and hospital and mortality outcomes. The EAVE sentinel system, which builds on the seasonal influenza vaccination study SIVE [12], allows rapid evaluation of any new vaccination (and antivirals). EAVE also provides information to help with targeting any available pandemic influenza vaccine at those who are considered to be at increased risk of serious illness or death from pandemic influenza infection e.g. those with any underlying medical conditions; those who may lack cross-reactivity from exposure to previous pandemics or vaccinations; and novel risk groups that are uniquely at risk because of a tropism exhibited by the virus [13]. For any new pandemic influenza vaccine, it may take some time until matched vaccines are available. Stockpiled pre-pandemic vaccine (e.g. H5, H7) for the UK population, 
recommended to be of sufficient quantities for those aged 16 or under and aged 65 or over could be used for early testing $[14,15]$.

The FLU-CATs (real time refinement and validation of criteria and tools used in primary care) study captures the criteria that allows validation of several triage tools in the early stage of an outbreak in readiness for deployment of these tools should surge occur [16]. During pandemics, surge in health-care demand can exceed capacity to provide normal standards of care. Healthcare workers who are unfamiliar with clinical assessment and admission decision-making may be asked to fulfil 'gatekeeper' roles. Triage tools may aid decisions in identifying people who are most likely to benefit from the higher levels of care that can only be provided in hospital. Anonymised details of patients with influenza-like-illness attending a study general practice will be shared with the study team. These details include: assessment, management, selected medical record excerpts, record hospital discharge data and death notices [16]. FLU-CATs activates during winter influenza seasons at a limited number of sites (5 to 20) to maintain study processes. This has allowed validation of the Department of Health Community Assessment Tools for use in children and adults presenting with seasonal influenza like illness

The PAINTED study (PAndemic INfluenza Triage in the Emergency Department) addresses the issue of needing to prioritise a potentially large number of patients with limited resources in the event of a pandemic [17]. Clinicians will need to identify patients at higher risk of poor outcomes, ideally with minimal or no supplementary testing. This study will use a standardised clinical assessment form to collect data on adults and children presenting to UK emergency departments in the event of a pandemic. Patients will be followed up at 30 days to identify those who have died or needed life-saving interventions and the information collected will be used to evaluate current, and develop new, triage methods. 
The UK Obstetric Surveillance System (UKOSS) influenza in pregnancy study will collect information on pregnant and postpartum women admitted to hospital with confirmed influenza infection [18]. Pregnant women are known to be at high risk of severe outcomes of a number of infections, and appeared disproportionately affected in the 2009 A/H1N1 influenza pandemic [18]. UKOSS will collect information on the management of women, focussing particularly on the role of extracorporeal membrane oxygenation (ECMO), and women will be followed up to pregnancy completion in order to collect information on both maternal and perinatal outcomes.

The multi-centre Adjuvant Steroids in Adults with Pandemic Influenza (ASAP) Trial will determine the role of corticosteroids in adults admitted to hospital with influenza infection. Corticosteroids were used widely during the 2009 A/H1N1 influenza pandemic, particularly for severely ill patients. However increased mortality was found in observational studies [19]. The ASAP trial will recruit 2200 participants by the end of the first wave of the next pandemic with trial results available to inform clinical practice before the start of the second wave [20].

The Real-time modelling of a pandemic influenza outbreak (RTM) study has been funded to advance the state of the art of real-time modelling of influenza epidemics and to provide a tool to monitor and predict the development of an ongoing pandemic outbreak in the UK. Outputs from the RTM study include: models to produce age and region-specific epidemic forecasts [21]; algorithms, building on the latest developments in statistical computation, to allow epidemic analyses to be updated in a timely fashion as the epidemic unfolds [22]; bespoke software and relevant training of Public Health England (PHE) staff.

\section{Role of the funding source}


The sponsor of the study had no role in study design, data collection, data analysis, data interpretation, or writing of the report. The corresponding author had full access to all the data in the study and had final responsibility for the decision to submit for publication.

\section{Hibernation challenges}

The need to "hibernate" studies while awaiting a pandemic is a feature of the pandemic influenza portfolio studies that has raised a number of issues. This reflects both the need to maintain interest and engagement of stakeholders (including those who initially agreed to host the research, policy makers and others), and changes to the landscape in terms of research regulations, changes in science and social changes.

\section{Maintaining engagement of research sites to ensure rapid activation}

Reduced research nurse availability in the event of a pandemic due to sickness absence and indirectly due to reallocation to clinical duties is a particular issue for several of the studies [17]). Furthermore, for the ASAP trial it is critical that hospital sites remain in a 'simmering' state of readiness for an extended period of time and that potential obstacles that might arise following activation and require 'time-costly' solutions are minimised [20]. For instance, there will be no opportunity to conduct and evaluate a pilot study once a pandemic arises. Instead, an activation simulation exercise was required which enabled the identification of stumblingblocks and refinement of trial processes, adding to trial readiness. One important issue highlighted was the need for 'backup' plans and pre-assigned 'deputy' roles (including for the Chief Investigator), as part of trial resilience [23].

The PIPS study was specifically designed to piggyback on existing infrastructure provided by the Health Survey for England (HSE) [24], an annual, nationally representative, household survey which collects health information and blood specimens throughout the year [9]. This 
study is therefore dependent on the long-term continuation of the HSE and its continuation during a pandemic.

The RTM study's prompt activation in the event of a pandemic depends on the continued commitment of relevant public bodies (e.g. PHE) and collaborators to provide ready access to the agreed available data.

\section{Approvals within a changing regulatory environment}

Studies report that research sites' research administration staff regularly attempt to close hibernated studies with researchers needing to repeatedly explain the hibernation status to NHS organisations that are unfamiliar with the concept and do not have systems in place to incorporate this form of research into their regulatory frameworks. The term 'hibernated' thus possibly represents a misnomer; such studies are perhaps better thought of as 'in progress, but awaiting activation of data collection'. There may be a need for new processes designed specifically for studies such as these.

\section{Dealing with changing technologies}

The PAINTED study was designed to recruit patients on the basis of clinical suspicion of pandemic influenza [17]. Recent advances in point of care technology mean that suspected seasonal influenza can be rapidly diagnosed or ruled out in the emergency department [25]. If applicable in a pandemic this would change the study population but would not undermine the need for the study or change the study design. An observational cohort study would still be needed to identify predictors of adverse outcome in diagnosed pandemic influenza (rather than suspected pandemic influenza), and therefore a modified PAINTED study with a different population would continue. Influenza serological assays require specialist expertise only available in selected laboratories that will have limited research capacity during a pandemic due to a substantial increase in workload. Maintaining expertise outside of these centres is 
necessary to ensure that researchers [9] can rapidly initiate testing of samples. Pilot work carried out in the PIPS study identified that establishing de-novo serological testing capacity led to unacceptable delays. In response to this finding, the NIHR is funding short laboratory placements in the WHO Collaborating Centre for Reference and Research on Influenza for laboratory staff to maintain a pool of expertise that can be rapidly deployed. The WHO collaborating centre will also have access to the novel virus and the first available serological assay so these links will support the study's rapid assay set up and deployment. Consideration is being given in how to best run rapid turn-around surveys. For example, the number of households that have solely mobile phones is increasing. Such households may differ from landline households on several demographic, and psychological levels. The FluTEST team is monitoring changes in the science of polling methodology and exploring the possible costeffectiveness of alternative approaches such as social media monitoring [26].

\section{Maintaining readiness of electronic platforms}

Rapid evolution of the IT landscape is inevitable so adequate resources for maintenance of such studies is imperative. The FLU-CATs study was established on an electronic Health Record (eHR) system (Vision, In Practice Systems Ltd) in partnership with the Clinical Practice Research Datalink [16]. The use of this platform has gradually decreased within the primary care community as web and cloud based systems have become more popular. To retain representative coverage in the UK the FLU-CATs study has been obliged to add an addition eHR solution which will sit on the EMIS Health eHR (Egton Medical Information Systems Ltd.). This will be rolled out in collaboration with the Royal College of General Practitioners Research and Surveillance Centre.

The PAINTED study has been designed to use paper records, in accordance with typical NHS emergency department practice at the time [17]. However, increasing numbers of NHS 
emergency departments are developing electronic data systems. The new Emergency Care Data Set (ECDS) has a research data item that allows identification of cases recruited to research and could be used to electronically flag cases included in the PAINTED study. Further pilot work is now required to determine whether and how the new ECDS and new emergency department data systems can be used to optimise data collection.

The UKOSS research platform switched from a paper-based reporting system to an electronic one during the hibernation period. Additional funding to activate the study to collect information on seasonal influenza management and outcomes in pregnancy has enabled the required programming to be undertaken to maintain the hibernated pandemic influenza study in readiness [18].

\section{Workforce issues during a pandemic}

The PIPS study is dependent on the continuation of the Health Survey for England during a pandemic and its ability to either maintain laboratory expertise in influenza serology or recruit staff with relevant experience. The data analysis protocols have been automated and could be run by alternative staff. A similar approach has been taken by the EAVE project whereby systems have been automated sufficiently for non-core staff to run the relevant syntax to extract and analyse data. For FLU-CATS, the study process relies on both continued activity by General Practitioners in Primary Care to continue their usual consultation activity for Influenza Like Illness using their usual Electronic Health Care Records (EHRs). The study runs on background processes that harvest information from these consultations. Substitution by locums will not inherently affect the study provided consultations continue and the EHR is used. The study research team is small, however the processes for data analysis have been archived and test activated against the current EHR systems, so could be run by substitute staff. For FLU-TEST, the open-access publication of the protocol and survey questions allows 
anyone to use the FluTEST items and analyse the resulting data. For ASAP all sites and participating research partners (including manufacturing units) have been requested to identify persons who can deputise for key research staff in the event of absence from work. There is also an internal Pandemic Plan to ensure the project can continue to deliver the trial, including a "co-Chief Investigator". PAINTED is currently exploring creating an electronic triage form for Emergency Department systems that could be used to collect electronic data for the study as part of routine clinical care. Furthermore, using record linkage to NHS Digital will allow the study to be undertaken with minimal research nurse support.

\section{Influenza pandemic preparedness and emerging infective/major incident}

\section{challenges}

This national portfolio of hibernated pandemic influenza studies is now mature, and, we believe, clearly illustrates the value of the UK's clinical research system and the potential for rapid research, as well as clinical and public health response, in a future pandemic. However, when and whether a future influenza pandemic occurs is clearly unpredictable. What is clearer, is that other emerging infective challenges will continue to occur. Can these studies act as a model for research in a future pandemic or emerging infection? Experience suggests they can as UKOSS was used to study Zika virus exposure during pregnancy [27].

Other hibernated studies could equally address research questions likely to be essential in any future epidemic or pandemic and not just those specific to influenza. For instance, the FLUCATS response to changes in the architecture of EHRs - the study tools could be used in either primary or secondary care settings for a variety of emerging diseases and deliberately/accidentally released agent (CBRN) (although the performance of the tool will vary with the population being triaged) [16]. PAINTED is focused on triage, given its importance in ensuring patients with suspected influenza are managed appropriately in a busy 
emergency department setting [17]. However, a robust triage process is likely to be important if any other emerging infection results in large numbers of patients seeking emergency care, in order to ensure that the health system can function. As departments continue to face capacity issues, exploring the role of triage in the event of other epidemic or pandemics would be valuable in assessing its effectiveness in times of severe stress on health systems.

The EAVE project has created a new sentinel system linking primary care data to RT-PCR swabs, stored serology and hospital and mortality outcomes. The project will allow for a rapid evaluation of any new vaccination irrespective of the infection it is developed to test against. Also, as noted above, serological analysis of stored samples on susceptibility is dependent on the provision of an assay that will allow this work to be undertaken. Experience of the last pandemic would indicate that there will likely be some delay in the development and deployment of such an assay. Prior agreement for access to the first assay approved for use would be important to maximise the study's benefit.

The PIPS project specimen and data collection could also be used for other epidemic/pandemic scenarios provided a serological marker of susceptibility/infection is available. Similarly, surveys using the FluTEST survey methodology could be used to monitor behaviour whatever the public health emergency (including CBRN risks).

The RTM system could also be exploited in other outbreaks and, in fact, could be regularly used outside a pandemic to anticipate the burden of seasonal influenza. This would also test the easiness of its implementation and give PHE staff the opportunity to develop their expertise. However, use on seasonal data would pose challenges around release of relevant data (e.g. on general practice consultations), whose access should be instead guaranteed in the midst of a pandemic. 
Irrespective of the emerging infection, trials will be needed - a network set up and ready to test one intervention versus standard care will be invaluable to fast track the evidence needed for clinical practice and the periodic activation exercise undertaken by the ASAP trial provides a model for this. Work has already begun to develop the ASAP Trial network for the broader purpose of determining the role of adjuvant corticosteroids in severe acute respiratory viral infections other than pandemic influenza.

\section{Lessons learned}

Although more information on project performance will be available once projects have been brought out of hibernation for testing, there have already been a number of lessons learned during the set-up phase. There may be a need for incremental modification in the event of changes in the architecture of EHRs. Approaches are needed to mitigate for reduced staff availability in the event of a pandemic due to sickness absence and indirectly due to reallocation to clinical duties e.g. pre-assigned 'deputy' roles and automation of processes. Commitment of relevant public bodies and collaborators e.g. to provide ready access to agreed available data should be maintained. A pool of expertise (beyond selected laboratories) that can be rapidly deployed is needed to carry out de-novo serological testing during pandemic. Organisational regulatory frameworks need to be flexible for studies that require long periods before activation. With technological advances for running rapid turn-around surveys, consideration should be given to alternative approaches that may be faster and cheaper

\section{Next steps}

Should the UK's portfolio of hibernated pandemic influenza studies provide a model for how we think about planning research in public health emergencies, including emerging infections, going forward? All too often researchers are in competition when trying to answer research questions rapidly in an emergency situation. The research response to the Ebola outbreak in 
West Africa was late and then inappropriately competitive resulting in several underpowered unsuccessful studies [28]. In contrast, the UK model allows for advance funding and planning of a complementary suite of studies, system testing (a test has been carried out in 2018 with reports compiled by the NIHR) and developing a collaborative network of researchers. Existing international networks e.g. the International Severe Acute Respiratory and Emerging Infection Consortium (ISARIC) [29] and the International Network of Obstetric Survey Systems (INOSS) [30] either are or could be primed and ready to go internationally with similar studies. The challenge going forward will be, however, for commercially funded studies not to compete for scarce "patient resources" and to fit within this framework to ensure the highest quality studies are conducted most expediently. With this in mind, is now the time for a national/international register of planned pandemic and emerging infection studies with agreements over priorities and co-enrolment and collaboration?

Whilst an influenza pandemic heads the UK National Risk Register of Civil Emergencies [31], large infectious outbreaks do not constitute the only public health risk. Research to improve care is also essential in other public health emergencies such as natural disasters and the deliberate release of biochemical weapons as part of terrorist attacks [8] [32]. Coordination across research and funding bodies (commercial, charity and government) will be required if expectations demanded by the public of the research community are to be met in response to public health emergencies. Is this the time to consider a new way of working together as part of emergency preparedness, including public engagement on the ethical questions that arise?

Contributors: MK and CS led the writing of this personal view. All authors made contributions to the writing and discussions on the scope of the personal view and critically reviewed revisions and the final paper. 
Conflicts of interest: SG is Chair of the NIHR Health Technology Assessment Clinical Evaluation and Trials Board and chaired the NIHR Pandemic Influenza Themed Call Board. All authors reports grants from NIHR, during the conduct of the study. DA reports grants from Public Health England, outside the submitted works. WSL's institution has received unrestricted investigator-initiated research funding from Pfizer for an unrelated research study in pneumonia where WSL is the chief investigator.

Acknowledgements: The authors would like to thank all the collaborators of this work. These studies were funded by grants from the National Institute for Health Research Health under its Pandemic Influenza Preparedness call. The views and opinions expressed therein are those of the authors and do not necessarily reflect those of the HS\&DR, HTA and Public Health programmes, NIHR, NHS or the Department of Health. 


\section{References}

1. Walley T, Davidson P. Research funding in a pandemic. Lancet 2010;375: 1063-5

2. Simpson CR, Ritchie LD, Robertson C, Sheikh A, McMenamin J. Effectiveness of $\mathrm{H} 1 \mathrm{~N} 1$ vaccine for the prevention of pandemic influenza in Scotland, UK: a retrospective observational cohort study. Lancet Infect Dis 2012; 12:696-702

3. Goodacre S, Challen K, Wilson R, Campbell M. Evaluation of triage methods used to select patients with suspected pandemic influenza for hospital admission: cohort study. Health Technol Assess. 2010;14:173-236

4. Killingley B, Greatorex J, Cauchemez S, et al. Virus shedding and environmental deposition of novel A (H1N1) pandemic influenza virus: interim findings. Health Technol Assess. 2010;14:237-354

5. Annane D, Antona M, Lehmann B, et al. Designing and conducting a randomized trial for pandemic critical illness: the $2009 \mathrm{H} 1 \mathrm{~N} 1$ influenza pandemic. Intensive Care Med. 2012;38:29-39

6. Rubin GJ, Amlôt R, Page L, Wessely S. Public perceptions, anxiety and behavioural change in relation to the swine flu outbreak: A cross-sectional telephone survey. BMJ 2009; 339: b2651

7. Nguyen-Van-Tam JS, Openshaw PJ, Hashim A, et al. Risk factors for hospitalisation and poor outcome with pandemic A/H1N1 influenza: United Kingdom first wave (May-September 2009). Thorax. 2010;65:645-5

8. Lurie N, Manolio T, Patterson AP, Collins F, Frieden T. Research as a part of public health emergency response. $N$ Engl J Med 2013; 368:1251-5

9. Fragaszy EB, Quinlivan M, Breuer J, et al. Population-level susceptibility, severity and spread of pandemic influenza: design of, and initial results from, a pre-pandemic and hibernating pandemic phase study using cross-sectional data from the Health Survey for England (HSE). Public Health Research, 2015; 3: 1-24

10. Rubin GJ, Potts HWW, Michie S. Likely uptake of swine and seasonal flu vaccines among healthcare workers. A cross-sectional analysis of UK telephone survey data. Vaccine 2011; 29: 2421-28.

11. Rubin GJ, Potts HWW, Michie S. The impact of communications about swine flu (influenza A H1N1v) on public responses to the outbreak: Results from 36 national telephone surveys in the UK. Health Technol Assess 2010; 14:183-266

12. Simpson CR, Lone N, Kavanagh K, et al. Seasonal influenza vaccine effectiveness (SIVE): an observational retrospective cohort study- exploitation of a unique community-based national-linked database to determine the effectiveness of the seasonal trivalent influenza vaccine. Health Serv Deliv Res 2013; 1(10) 
13. Simpson CR, Lone N, McMenamin J, et al. Early estimation of pandemic influenza Antiviral and Vaccine Effectiveness (EAVE): use of a unique community and laboratory national data-linked cohort study. Health Technol Assess 2015;19:1-32

14. Scientific Pandemic Influenza Group on Modelling SPI-M Modelling Summary Accessed from:

https://assets.publishing.service.gov.uk/government/uploads/system/uploads/attachme nt_data/file/756738/SPI-M_modelling_summary_final.pdf [Last Accessed November 2018]

15. Baguelin M, Flasche S, Camacho A, et al. Assessing Optimal Target Populations for Influenza Vaccination Programmes: An Evidence Synthesis and Modelling Study. PLoS Medicine 2013; 10, e1001527

16. Venkatesan S, Myles PR, McCann G, et al. Development of processes allowing near real-time refinement and validation of triage tools during the early stage of an outbreak in readiness for surge: the FLU-CATs Study. Health Technol Assess 2015;19:1-132

17. Goodacre S, Irving A, Wilson R, Beever D, Challen K. The PAndemic INfluenza Triage in the Emergency Department (PAINTED) pilot cohort study. Health Technol Assess, 2015; 19: 1-69

18. Knight M, Brocklehurst P, O’Brien P, Quigley MA, Kurinczuk JJ. Planning for a cohort study to investigate the impact and management of influenza in pregnancy in a future pandemic. Health Serv Deliv Res 2015; 3.6

19. Rodrigo C, Leonardi-Bee J, Nguyen-Van-Tam JS, Lim WS. Effect of corticosteroid therapy on influenza-related mortality: a systematic review and meta-analysis. $J$ Infect Dis 2015;212:183-9

20. Lim WS, Brittain C, Duley L, et al. Blinded randomised controlled trial of low-dose Adjuvant Steroids in Adults admitted to hospital with Pandemic influenza (ASAP): a trial 'in hibernation', ready for rapid activation. Health Technol Assess 2015;19:1-78

21. Birrell PJ, Zhang X-S, Pebody RG, Gay N, De Angelis D. Reconstructing a spatially heterogeneous epidemic: Characterising the geographic spread of 2009 A/H1N1 infection in England. Scientific Reports 2016; 11:29004

22. Birrell PJ, Pebody RG, Charlett A, Zhang X-S \& De Angelis D. Real-time modelling of a pandemic influenza outbreak. Health Technol Assess 2017;21: 1-118

23. Lim WS, Meakin G, Brittain C, Bewick T, Duley L. Improving readiness for recruitment through simulated trial activation: the Adjuvant Steroids in Adults with Pandemic influenza (ASAP) trial. Trials 2017;18:546

24. Fuller E, Mindell J, Prior G (eds) Health Survey for England 2016, London: NHS Digital 2017 
25. Davis S, Allen AJ, O'Leary R, et al. Diagnostic accuracy and cost analysis of the Alere $^{\mathrm{TM}} \mathrm{i}$ Influenza A\&B near-patient test using throat swabs. J Hosp Infect 2017;97:301-309

26. Rubin GJ, Bakhshi S, Amlôt R, Fear N, Potts H, Michie S. The design of a survey questionnaire to measure perceptions and behaviour during an influenza pandemic: The Flu Telephone Survey Template (FluTeST). Health Services and Delivery Research 2014; 2(41)

27. NPEU. Pregnancy outcomes in mothers with a history of travel to a country with active Zika transmission. https://www.npeu.ox.ac.uk/ukoss/current-surveillance/zikavirus [Last accessed 9.4.2018]

28. Thielman NM, Cunningham CK, Woods C, Petzold E, Sprenz M, Russell J. Ebola clinical trials: Five lessons learned and a way forward. Clin Trials 2016;13:83-6

29. International Severe Acute Respiratory and Emerging Infection Consortium https://isaric.tghn.org [Last accessed 9.3.2018]

30. NPEU. International Network of Obstetric Survey Systems https://www.npeu.ox.ac.uk [Last accessed 9.3.2018]

31. UK National Risk Register of Civil Emergencies, Cabinet Office, 2015 https://www.gov.uk/government/publications/national-risk-register-of-civilemergencies-2017-edition

32. Levine AC, Teicher C, Aluisio AR, et al. Regional Anesthesia for Painful Injuries after Disasters (RAPID): study protocol for a randomized controlled trial. Trials 2016;17:542 
Table 1 National Institute of Health Research Pandemic Influenza Preparedness Portfolio

\begin{tabular}{|c|c|c|c|}
\hline Project & Lead Applicant and Institute & Purpose of study & Link \\
\hline $\begin{array}{l}\text { The population-level } \\
\text { susceptibility, severity and spread } \\
\text { of pandemic influenza study } \\
\text { (PIPS) }\end{array}$ & $\begin{array}{l}\text { Professor Andrew Hayward, } \\
\text { University College London }\end{array}$ & $\begin{array}{l}\text { The PIPS study developed a system to rapidly assess real-time } \\
\text { community-level susceptibility and spread of infection and illness in } \\
\text { the event of a pandemic. It achieved this by adding additional } \\
\text { questions and specimen collection to the Health Survey for England, } \\
\text { an annual, nationally-representative survey that recruits participants } \\
\text { throughout the year. }\end{array}$ & $\begin{array}{l}\text { https://www.ncbi.nl } \\
\text { m.nih.gov/books/N } \\
\text { BK299604/pdf/Boo } \\
\text { kshelf_NBK299604. } \\
\text { pdf }\end{array}$ \\
\hline $\begin{array}{l}\text { Flu Telephone Survey Template } \\
\text { study (FluTEST) }\end{array}$ & $\begin{array}{l}\text { Dr James Rubin, King's College } \\
\text { London }\end{array}$ & $\begin{array}{l}\text { The preliminary work of FluTEST involved developing, testing and } \\
\text { publishing a broad range of survey questions that can be used with } \\
\text { confidence in the next pandemic to identify public knowledge, } \\
\text { attitudes and behaviour. During a pandemic we will support the UK } \\
\text { Department of Health and Social Care in deploying these items and } \\
\text { interpreting the results. }\end{array}$ & $\begin{array}{l}\text { https://www.ncbi.nl } \\
\text { B.nih.gov/books/N } \\
\text { BK263566 }\end{array}$ \\
\hline $\begin{array}{l}\text { Early estimation of pandemic } \\
\text { influenza Antiviral and Vaccine } \\
\text { Effectiveness (EAVE): use of a } \\
\text { unique community and laboratory } \\
\text { national linked dataset }\end{array}$ & $\begin{array}{l}\text { Professor Colin Simpson, The } \\
\text { University of Edinburgh }\end{array}$ & $\begin{array}{l}\text { The EAVE project created a new sentinel system that links primary } \\
\text { care data to RT-PCR swabs, stored serology and hospital and } \\
\text { mortality outcome data. The project allows for a rapid evaluation of } \\
\text { any new vaccination (and antivirals) and provides information to help } \\
\text { with targeting any available pandemic influenza vaccine at those who } \\
\text { are considered to be at increased risk of serious illness or death from } \\
\text { pandemic influenza infection. }\end{array}$ & $\frac{\text { https://www.ncbi.nl }}{\underline{\text { B.nih.gov/books/N }}}$ \\
\hline
\end{tabular}




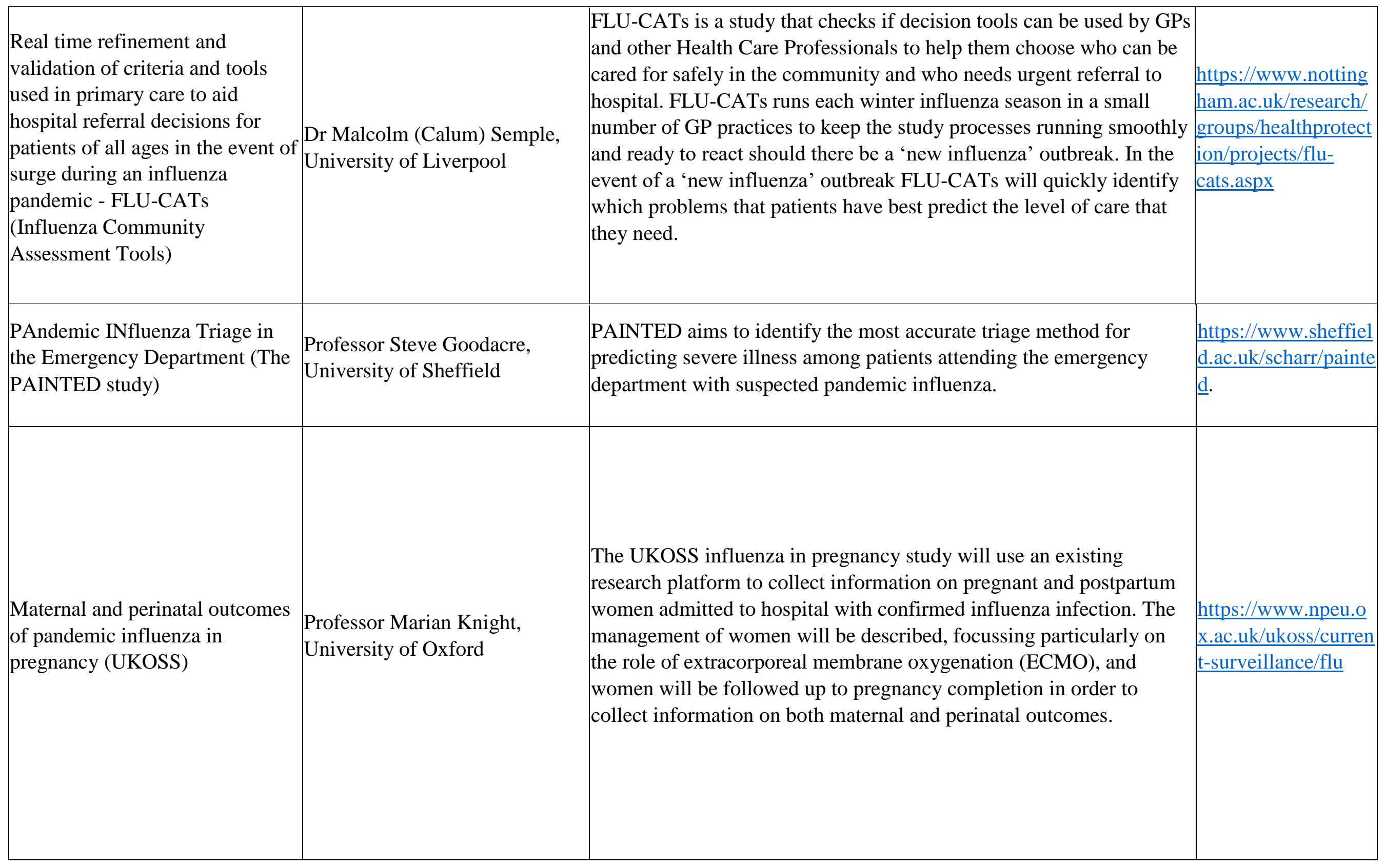




\begin{tabular}{|c|c|c|c|}
\hline $\begin{array}{l}\text { Multi-centre Adjuvant Steroids in } \\
\text { Adults with Pandemic Influenza } \\
\text { (ASAP) Trial }\end{array}$ & $\begin{array}{l}\text { Professor Wei Shen Lim, } \\
\text { Nottingham University Hospitals }\end{array}$ & $\begin{array}{l}\text { The ASAP trial is a multicentre ( }>40 \text { sites) blinded randomised } \\
\text { controlled clinical trial to determine if low dose corticosteroids } \\
\text { (dexamethasone } 6 \mathrm{mg} \text { started within } 24 \text { hours of admission once a day } \\
\text { for } 5 \text { days), in addition to standard care, is associated with a lower risk } \\
\text { of death or admission to intensive care, compared to placebo. }\end{array}$ & https://asaptrial.org/ \\
\hline $\begin{array}{l}\text { Real-time Modelling of a } \\
\text { Pandemic Influenza Outbreak } \\
\text { (RTM) }\end{array}$ & $\begin{array}{l}\text { Dr Daniela De Angelis } \\
\text { University of Cambridge }\end{array}$ & $\begin{array}{l}\text { The real time model (RTM) project advances the state of art for real } \\
\text { time pandemic modelling by developing an existing model used to } \\
\text { reconstruct the } 2009 \text { H1N1 pandemic on the basis of realistic } \\
\text { epidemic surveillance data collected. The work for the RTM has } \\
\text { already been carried out and has resulted in a new monitoring tool that } \\
\text { allows to: capture spatial variation in influenza transmission; uses } \\
\text { efficient computational algorithms for the provision of timely } \\
\text { statistical estimates and predictions; and incorporates the above into } \\
\text { freely available software. The tool is now available to Public Health } \\
\text { England for use in the advent of a pandemic, and key staff has been } \\
\text { trained in its use, supported by collaborators at the University of } \\
\text { Cambridge to deal with workforce shortage during a pandemic. The } \\
\text { real time model has been tested in the monitoring of the } 2017 / 2018 \\
\text { seasonal influenza and ready to be used in the current season to } \\
\text { estimate infection and clinical attack rates and to predict timing and } \\
\text { magnitude of the peak influenza activity. }\end{array}$ & $\frac{\text { https://www.ncbi.nl }}{\text { m.nih.gov/books/N }}$ \\
\hline
\end{tabular}

